\title{
Barrier-repair prescription moisturizers: Do we really need them? Facts and controversies.
}

\author{
Ronni Wolf \\ The Dermatology Unit, Kaplan Medical Center, Rechovot, Israel. Affiliated to The School of Medicine, \\ Hebrew University and Hadassah Medical Center, Jerusalem, Israel. \\ Lawrence Parish \\ Thomas Jefferson University
}

Follow this and additional works at: https://jdc.jefferson.edu/dcbfp

Part of the Dermatology Commons

Let us know how access to this document benefits you

\section{Recommended Citation}

Wolf, Ronni and Parish, Lawrence, "Barrier-repair prescription moisturizers: Do we really need them? Facts and controversies." (2013). Department of Dermatology and Cutaneous Biology Faculty Papers. Paper 31.

https://jdc.jefferson.edu/dcbfp/31

This Article is brought to you for free and open access by the Jefferson Digital Commons. The Jefferson Digital Commons is a service of Thomas Jefferson University's Center for Teaching and Learning (CTL). The Commons is a showcase for Jefferson books and journals, peer-reviewed scholarly publications, unique historical collections from the University archives, and teaching tools. The Jefferson Digital Commons allows researchers and interested readers anywhere in the world to learn about and keep up to date with Jefferson scholarship. This article has been accepted for inclusion in Department of Dermatology and Cutaneous Biology Faculty Papers by an authorized administrator of the Jefferson Digital Commons. For more information, please contact: JeffersonDigitalCommons@jefferson.edu. 
As submitted to:

Clinics in Dermatology

And later published as:

Barrier-repair prescription moisturizers: do we really need them?

Facts and Controversies.

2013, Nov-Dec, Volume 31, Issue 6, pp. 787-91.

DOI: 10.1016/j.clin dermatol.2013.05.018.

Ronni Wolf, MD*; Lawrence Charles Parish MD, MD (Hon $)^{* *}$

From:

* The Dermatology Unit, Kaplan Medical Center, Rechovot, Israel. Affiliated to The School of Medicine, Hebrew University and Hadassah Medical Center, Jerusalem. ** Department of Dermatology and Cutaneous Biology, Jefferson Medical College of Thomas Jefferson University, Philadelphia, PA, USA 
Address for Correspondence: Ronni Wolf, MD, Dermatology Unit, Kaplan Medical

Center, Rehovot 76100 , Israel. Fax +972-9-9560978. E-mail: wolf_r@ @etvision.net.il 


\section{Abstract}

There is now scientific evidence of genetically driven skin barrier anomalies in atopic patients. These barrier anomalies facilitate sustained antigen ingress through the defective barrier, which can bring about a Th2-dominant response. It enhances the transepidermal water loss, resulting in dry skin and leading to the release of pre-formed pro-inflammatory cytokines and to a cascade of events ending up in inflammation.

There is scientific evidence indicating that skin barrier dysfunction plays a prominent role in the development and perpetuation of atopic dermatitis (AD), the "outside-to-inside" view of $\mathrm{AD}$ pathogenesis. Moisturizers have a pivotal role in improving and maintaining the skin barrier function and in reducing skin susceptibility to irritants. These emollients are a standard of care, the mainstay of AD therapy, and useful for both prevention and maintenance therapy.

Regular use of emollients has a short- and long-term steroid-sparing effect in mild-tomoderate AD. They should be recommended in adequate amounts and used liberally and frequently in atopic patients. Recently, "Barrier-repair therapeutics" has entered the market, generally as devices, requiring prescriptions (510k-cleared moisturizers). Although this new class of moisturizers has shown excellent performance, efficacy, and safety, we could raise questions about their superiority over a well-crafted traditional, petrolatum-based OTC emollient, as we have been unable to find any scientific evidence to support this claim. In addition, there is currently no definition for "barrier-repair products," raising the additional question of whether such presently available products could justify such a category. 


\section{The "outside-to-inside" view of atopic dermatitis and its therapeutic implications}

Until recently, it was thought that the pathogenic mechanism of atopic dermatitis (AD) was initiated by immune dysfunction leading to Th1/Th2 cell dysregulation, IgE production, mast cell hyperactivity, and dendritic cell signaling. Although both defective epidermal permeability that causes dry skin and leads to a predisposition to secondary infection are well-recognized features of $\mathrm{AD}$, these conditions have been widely assumed to reflect downstream consequences of a primary immunologic abnormality (the historical "inside-tooutside" view of AD pathogenesis). Accumulating evidence, however, now suggests that a primary defect in the stratum corneum may play a key role not only in initiating but also in perpetuating AD. This dysfunction of the barrier leads to the release of pre-formed proinflammatory cytokines and sets into motion a cascade of events that provoke and sustain disease activity (the "outside-to-inside" view of AD pathogenesis).

Two main mechanisms account for the outside-to-inside direction of events, that is to say, from the impaired barrier to the development of the inflammation. One is the release of a cytokine cascade that up-regulates lipid synthesis, lamellar body secretion, and epidermal proliferation aimed at the restoration of the barrier function and the initiation of inflammation. The other is the penetration of irritants, allergens, haptens, superantigens, and bacterial toxins.

Awareness of the primary role of the stratum corneum in the pathogenesis of $\mathrm{AD}$, as well as in other inflammatory diseases, has opened up new therapeutic options and a new therapeutic concept: to treat not only the inflammation but also to restore the barrier. The historical origins of this concept derive from the numerous, innovative works of Elias and co-workers, which have been well presented in a 2001 paper with the catchy title, "Does the tail wag the dog?" ${ }^{1}$ A further step in this direction was the development of a new pharmacologic approach aimed at barrier repair. It involves the delivery of balanced proportions of stratum corneum-specific lipids to assist in correcting this epidermal barrier dysfunction. 
In a state of physiologic balance, the approximate proportions of the lipid component are predominantly composed of $50 \%$ ceramides, $25 \%$ cholesterol, and $10-20 \%$ free fatty acids. In $\mathrm{AD}$, there is a decrease in all three key lipids, especially ceramides, which are found in both affected and non-affected skin. A lipid imbalance and inadequate amounts of ceramides contribute to defective formation of the corneocyte lipid envelope and lipid mortar, which correlate with increased transepidermal water loss and enhanced barrier permeability ${ }^{2}$.

These scientific findings have led to a second look at the use of moisturizers.

There has been a shift from traditional moisturizers towards ceramide-dominant physiological lipid-base barrier-repair topical emulsions. According to several reports ${ }^{2}$, these formulations focus on physiologic lipid replacement therapy, particularly ceramides, to restore the normal balance of the epidermal barrier. Compared with other emollients that form a more superficial occlusive barrier (e.g., petrolatum), ceramide-dominant moisturizers are thought to permeate the stratum corneum and to be synthesized in the keratinocytes, processed in the lamellar bodies, and secreted back into the stratum corneum, where they become a part of the dermal matrix.

In an extensive review by Elias ${ }^{3}$, the developer of barrier-repair therapeutics, he states: “Typical emollient moisturizers, even if they provide some temporary relief through moisturization, actually degrade, rather than improve, barrier function, and by providing partial relief, they actually delay barrier repair." This, he claims, is in contrast to the use of corrective barrier repair creams that contain "sufficient quantities of all three key lipids that mediate barrier function (i.e., cholesterol, free fatty acids, and ceramides)..."

\section{0(k)-cleared prescription moisturizers}

These barrier-repair products to which Elias is referring are technically different from traditional over-the-counter (OTC) nonprescription moisturizers, because the Food and Drug 
Administration (FDA) has approved them as 510(k) devices. The 510(k) device approval process was originally developed to ensure the safety of equipment with an on/off switch. A device that reaches market via the $510(\mathrm{k})$ process is not considered to be "approved" by the FDA, but it can still be marketed and sold in the United States. Such products are generally referred to as "cleared" or "510(k)-cleared" devices, where the focus is on safety as opposed to efficacy. Although creams are not traditionally thought of as "devices," these topical agents have received 510(k) approval, because they induce a physical change in the skin, specifically, an increase in skin hydration resulting from a decrease in the transepidermal water loss (it is important to note that OTC moisturizers should have exactly the same effect, but they have not been registered as devices). Various formulations that produce barrier repair by different mechanisms currently have 510(k) approval as prescription drugs.

\section{Ceramide-dominant physiological lipid-base barrier-repair products.}

"EpiCeram® Skin Barrier Emulsion" can be considered as the prototype of a ceramidedominant physiological lipid-base barrier-repair product. According to Elias' description, it is made up of ceramides, free fatty acids, and cholesterol in what is labeled "optimized proportions (i.e., a 3:1:1 molar ratio)" The product obtained marketing clearance from the FDA in April 2006. What is interesting about this moisturizer is that it had originally been an OTC product called Triceram ${ }^{\circledR}$ and was subsequently designated as a prescription product called Epiceram ${ }^{\circledR}$, with no known changes having been made in its chemical structure.

Chamlin and colleagues ${ }^{4}$ published a letter of correspondence entitled "The initial promising results of a phase 1 trial of a ceramide-dominant, barrier-repair moisturizer in childhood atopic dermatitis." The final results of this trial then appeared as an original report ${ }^{5}$. This modest study was performed on 24 children who continued their prior therapy for stubbornto-recalcitrant $\mathrm{AD}$ with the only change, being the substitution of their usual moisturizer with 
the barrier-repair emollient. These authors reported impressive results. The severity scoring of $\mathrm{AD}$ (SCORAD) values improved significantly in 22 of the children by 3 weeks and in all 24 of them by 21 weeks. Transepidermal water loss levels (TEWL), which were elevated over involved and uninvolved areas at entry, decreased in parallel with SCORAD scores and continued to decline even after the SCORAD scores plateaued. In conclusion: "A ceramidedominant, barrier-repair emollient represents a safe, useful adjunct to the treatment of childhood AD”.

In a five-center, investigator-blinded, randomized trial ${ }^{6}$, EpiCeram ${ }^{\circledR}$ was compared to a midpotent steroid (Cutivate ${ }^{\circledR}$ (fluticasone ) ) cream in 121 moderate-to-severe AD patients, aged six months to 18 years. EpiCeram ${ }^{\circledR}$ reduced clinical disease severity, decreased pruritus, and improved sleeping habits at both 14 and 28 days after initiation of therapy. Although the fluticasone-treated group showed significantly greater improvement at 14 days, SCORAD, pruritus and sleeping habit scores for EpiCeram did not differ significantly from the fluticasone-treated group by 28 days. The conclusion of the authors was: " the ceramidedominant, physiological-lipid based formulation could represent an effective stand-alone or ancillary therapy for many pediatric patients with $\mathrm{AD} "$. What is missing is a study with a control group of age-matched pediatric AD patients who were treated solely with OTC moisturizers.

In another 5-center, open-label, interventional study ${ }^{7}$, a ceramide-dominant, physiologic lipid-based emulsion (EpiCeram ${ }^{\circledR}$ ) was evaluated for three weeks either as monotherapy or in combination with another $\mathrm{AD}$ treatment in 207 patients with mild-to-moderate $\mathrm{AD}$. Overall, approximately one-half of the subjects achieved success with investigator global assessment (clear or almost clear investigator global assessment scores) after three weeks of treatment. A large proportion of subjects (75\%) and investigators (for $77 \%$ of the subjects) 
reported satisfaction after three weeks of treatment. Unique to this study was the direct involvement of subjects in deciding whether to use an additional treatment in combination with the tested moisturizer. The majority of subjects $(71 \%)$ chose not to add the prescribed medication to the ceramide-dominant, physiologic lipid-based product during the three-week study period. In conclusion: "...the product was shown to be an effective agent, with or without additional topical therapy, to provide good clinical efficacy and high levels of investigator and patient satisfaction for many patients with mild-to-moderate AD."

The 3-month- to 16-year-old subjects who had been evaluated in the study underwent a subgroup analysis ${ }^{8}$ and the results indicated : "a large percentage of subjects (71\%) reported satisfaction with clinical results". Again, there was no comparison of the results with a matched control group treated with OTC moisturizers.

Subsequent to the claims that EpiCeram ${ }^{\circledR}$ is in a class of its own as a stand-alone moisturizer, being superior, came a paper ${ }^{9}$ comparing EpiCeram ${ }^{\circledR}$ with another newly developed hyaluronic acid-based foam among 30 subjects with mild dermatitis and 30 subjects with moderate dermatitis. It was an investigator-blinded and split-body studies in which the patients with moderate dermatitis were asked to use additionally a triamcinolone acetonide $0.1 \%$ cream. The authors concluded: "both formulations achieved statistically significant improvement in all clinical signs and symptoms of $\mathrm{AD}$ by week 4; however, the hyaluronic acid foam achieved statistically significant improvement in overall dermatitis severity by week 2, whereas the ceramide-containing emulsion cream did not. Could this indicate that there might be a chink in the armor??

For the record, there are at least 3 other products approved as $510(\mathrm{k})$ medical device creams that have become available over the past several years and cleared by the FDA for the treatment of $\mathrm{AD}$. One of those 3 products is Atopiclair ${ }^{\circledR}$, a nonsteroidal multicomponent 
product in which some key ingredients are mixed together with oil in water emulsion. Its beneficial effects have been proven in several impressive studies. We found four such multicenter, randomized, vehicle-controlled clinical studies that evaluated it. The first ${ }^{10}$ comprised 218 adults with mild-to-moderate $\mathrm{AD}$, who administered the test cream or vehicle cream 3 times per day to affected areas and those areas prone to be affected. The primary endpoint of the study was the score of the Eczema Area and Severity Index (EASI) at day 22. The results showed that the cream was statistically more effective than vehicle in all outcomes. As could be expected, the authors' conclusions were: “...the cream is rapidly effective and safe as a non-steroidal monotherapy in mild to moderate $\mathrm{AD}$, and it may be useful as a topical steroid sparing agent”.

The third of the four multicenter studies that we found ${ }^{12}$ was a randomized, double-blind, vehicle-controlled trial comprised of 142 patients aged 6 months to 12 years, whose treatment consisted of applying either the test substance or the vehicle 3 times per day. The primary endpoint for efficacy was the Investigator's Global Assessment (IGA) score for AD at day 22. The secondary endpoints were patient/caregiver's assessment of pruritus, onset, duration of itch relief, the EASI score, and the need for rescue medication in the event of an AD flare. The test substance (Atopiclair $®$ ) was found to be significantly more effective $(\mathrm{P}<.0001)$ than the vehicle cream for the primary endpoint and for all the secondary endpoints in this cohort of infants and children.

The fourth multicenter double-blind, randomized, vehicle-controlled study on 60 pediatric patients $^{13}$ also showed a significant difference between the tested product (Atopiclair ${ }^{\circledR}$ ) and the vehicle. This study consisted of a treatment period of 43 days. The test substance showed $80 \%$ improvement in the IGA score at day 22 , compared with a score of $26.3 \%$ for the vehicle. This statistical significance was sustained until the end of the study. The authors' 
conclusions were that the product "may be considered as one of the available regimens effective in the treatment of mild-to-moderate $\mathrm{AD}$ in children and adolescents". An OTC moisturizer control group was again conspicuously missing.

The second prescription device

cream, Mimyx ${ }^{\circledR}$, contains a unique lamellar matrix of pharmaceutical emollient substances, including palmitoylethanolamine (PEA). PEA is a cannabinoid agonist that has antiinflammatory, antipruritic features and the capacity to inhibit mast cell activation. We are aware of at least one very large, multinational, multicenter, non-controlled prospective study ${ }^{14}$ in which data on the effect of Mimyx ${ }^{\circledR}$ on 2456 patients from 525 centers were analyzed. The study showed substantial relief of objective and subjective manifestations of AD. The intensities of symptoms were reduced (with a combined score reduction of $58 \%$ in the entire population), previously used topical corticosteroids were discontinued by $56 \%$ of all patients, and the average weekly application rate decreased by $62 \%$.

The third prescription topical cream is the lipid-rich Eletone ${ }^{\circledR}$ emulsion. An investigatorblinded bilateral study ${ }^{15}$ compared Eletone ${ }^{\circledR}$ emulsion with pimecrolimus cream $1 \%$ among 20 patients with mild to moderate $\mathrm{AD}$. The participants applied pimecrolimus cream twice daily to a target lesion on one side of the body and also applied a topical medical device cream three times daily on a symmetrical target lesion on the opposite side of the body for four weeks. After the 4-week study period, 15 of 20 (75\%) sites of both treatments were rated "clear" (0) or "almost clear" (1) by the Physician Global Assessment (PGA). Overall, there was no statistically significant difference in PGA scores between the treatment sites throughout the study ( $\mathrm{p}=0.8236)$. The authors concluded: "A lipid-rich, non-steroidal, topical medical device cream was as effective in improving the AD symptoms as pimecrolimus cream." 


\section{Prescription moisturizers versus OTC moisturizers}

All of the above-cited studies demonstrated that each tested product is safe and effective in treating $\mathrm{AD}$, either as monotherapy or as adjuvant treatment together with other treatments. Some of them compared the product to steroids, some to a topical immunomodulator, and others to vehicle alone. Even if they achieve the highest marks for objectivity, quality, and clinical value, it is impossible for them to claim that any of the prescription device moisturizers are superior to ordinary OTC moisturizers, because none of them compared the tested product to an ordinary non-prescription OTC emollient/moisturizer, which is inarguably much less expensive.

There are, however, at least 2 other studies that did compare prescription moisturizers with OTC emollients. One of them ${ }^{16}$ compared a prescription moisturizer (Mimyx ${ }^{\circledR}$ ) to a mineral oil, petrolatum, and paraffin OTC moisturizer that had been commercially available for over 100 years. This investigator-blinded research utilized a split-body model in 60 subjects (30 with mild eczema, 30 with moderate eczema) to examine effect of the two products in the treatment of eczema of the arms or legs. The subjects with mild eczema were asked to use the OTC moisturizer twice daily to the randomized right or left target limb and a prescription device to the other randomized target limb. In addition, 30 subjects with moderate eczema were asked to use the OTC moisturizer and $0.1 \%$ triamcinolone acetonide cream (TAC) twice daily to the randomized limb and a prescription device and TAC. All subjects with mild and moderate eczema were rated as being clear by the dermatologist investigator at the end of the study. Each target site limb was evaluated separately, and at no time point during the study (i.e., at week 1,2 , or 4) were there any statistically significant differences between the OTC and device moisturizer. The subject assessments also showed no statistically significant differences between the OTC and device moisturizer at any time point. The investigator 
concluded that parity was established in the treatment of mild-to-moderate eczema with both products that showed excellent results.

The other study that we found ${ }^{17}$ compared the clinical efficacy of two prescription moisturizers and one petrolatum-based skin protectant moisturizer that had been on the market for many years as an OTC product (OTC-Pet). Thirty-nine patients aged 2-17 years with mild-to-moderate $\mathrm{AD}$ were randomized 1:1:1 to receive one of three treatments with instructions to apply the treatment, three times daily for three weeks. Evaluations were carried out at baseline, day 7, and day 21, and the following were measured: IGA (0-4), body surface area (BSA) involvement (0-100), investigator's global assessment of improvement (IGAI, 0-6), eczema area and severity index (EASI, 0-72), and a $100 \mathrm{~mm}$ visual analog scale (VAS) for itch intensity. The final results showed no significant difference for any of the measured parameters between the three groups on day 21. OTC-Pet demonstrated no significant difference in clinical efficacy from prescription devices moisturizers. The demonstration that the clinical success of OTC-Pet is at least as good as the clinical success of prescription device moisturizers suggests that the clinical trial results presented by the manufacturers of one agent need to be interpreted as representing the performance of that single agent in isolation and not as testimony to its superiority over any other prescription agents or OTC products.

\section{What should we recommend to our patients?}

If there is any scientific, evidence-based support for the superiority of the prescription device moisturizers over well-crafted, traditional, petrolatum-based OTC emollients, we could not find it. The obvious $\$ 64,000$ question would be, what should we recommend to our patients? It seems that this pressing question will have to remain unanswered until more convincing evidence-based and unbiased information becomes available. 


\section{Moisturizers for AD: Facts and controversies}

$\checkmark$ There is now scientific evidence of genetically driven skin barrier anomalies in atopic patients.

$\checkmark$ These barrier anomalies facilitate sustained antigen ingress through the defective barrier which can bring about a Th2-dominant response. It enhances the transepidermal water loss, resulting in dry skin and leading to the release of preformed pro-inflammatory cytokines and to a cascade of events ending up in inflammation.

$\checkmark$ There is scientific evidence indicating that skin barrier dysfunction plays a prominent role in the development and perpetuation of $\mathrm{AD}$, the "outside-to-inside" view of $\mathrm{AD}$ pathogenesis.

$\checkmark$ Moisturizers have a pivotal role in improving and maintaining the skin barrier function and reducing skin susceptibility to irritants ${ }^{18}$.

$\checkmark$ Emollients are a standard of care, the mainstay of AD therapy, and useful for both prevention and maintenance therapy ${ }^{18}$.

$\checkmark$ Regular use of emollients has a short- and long-term steroid-sparing effect in mild-tomoderate AD.

$\checkmark$ Emollients should be recommended in adequate amounts and used liberally and frequently in atopic patients.

$\checkmark$ The type of emollient best suited for this approach has not yet been delineated. 
$\checkmark$ "Barrier-repair therapeutics" has recently entered the market as prescription 510kcleared moisturizers.

$\checkmark$ Although this new class of moisturizers has shown excellent performance, emphasizing efficacy and safety, there is no evidence to support their superiority over a well-crafted traditional, petrolatum-based OTC emollient.

$\checkmark$ There is currently no definition for "barrier-repair products." It is unclear, if presently available products justify such a category. The issue is even more cloudy with the term "moisturizer," used for products which do not moisturize. This term was actually invented by Madison Avenue marketeers for promoting the simplistic idea that moistening the skin will keep it soft and flexible; however, there is currently no medical definition of these so called moisturizers or emollients.

$\checkmark$ The approval of emollients, which do not contain demonstrably active ingredients different from "ordinary" emolients, as prescription devices seems to be a technicality that confuses the issue. 
Reference List

1 Elias PM, Feingold KR. Does the tail wag the dog? Role of the barrier in the pathogenesis of inflammatory dermatoses and therapeutic implications. Arch Dermatol. 2001;137:1079-1081.

.2 Sajic D, Asiniwasis R, Skotnicki-Grant S. A look at epidermal barrier function in atopic dermatitis: physiologic lipid replacement and the role of ceramides. Skin Therapy Lett. 2012;17:6-9.

.3 Elias PM. An Appropriate Response to the Black-Box Warning: Corrective, Barrier Repair Therapy in Atopic Dermatitis. Clin Med Dermatol. 2009;2:1-3.

.4 Chamlin SL, Frieden IJ, Fowler A, Williams M, Kao J, Sheu M, et al. Ceramidedominant, barrier-repair lipids improve childhood atopic dermatitis. Arch Dermatol. 2001;137:1110-1112.

.5 Chamlin SL, Kao J, Frieden IJ, Sheu MY ,Fowler AJ, Fluhr JW, et al. Ceramidedominant barrier repair lipids alleviate childhood atopic dermatitis: changes in barrier function provide a sensitive indicator of disease activity. J Am Acad Dermatol. 2002;47:198-208.

.6 Sugarman JL, Parish LC. Efficacy of a lipid-based barrier repair formulation in moderate-to-severe pediatric atopic dermatitis. J Drugs Dermatol. 2009;8:11061111.

.7 Kircik LH, Del Rosso JQ, Aversa D. Evaluating Clinical Use of a Ceramide-dominant, Physiologic Lipid-based Topical Emulsion for Atopic Dermatitis. J Clin Aesthet Dermatol. 2011;4:34-40. 
8 Kircik LH, Del Rosso JQ. Nonsteroidal treatment of atopic dermatitis in pediatric patients with a ceramide-dominant topical emulsion formulated with an optimized ratio of physiological lipids. J Clin Aesthet Dermatol. 2011;4:25-31.

9 Draelos ZD. A clinical evaluation of the comparable efficacy of hyaluronic acid-based foam and ceramide-containing emulsion cream in the treatment of mild-tomoderate atopic dermatitis. J Cosmet Dermatol. 2011;10:185-188.

.10 Abramovits W, Boguniewicz M. A multicenter, randomized, vehicle-controlled clinical study to examine the efficacy and safety of MAS063DP (Atopiclair) in the management of mild to moderate atopic dermatitis in adults. J Drugs Dermatol. 2006;5:236-244.

.11 Abramovits W, Hebert AA, Boguniewicz M, Kempers SE, Tschen E, Jarratt MT, et al. Patient-reported outcomes from a multicenter, randomized, vehicle-controlled clinical study of MAS063DP (Atopiclair) in the management of mild-to-moderate atopic dermatitis in adults. J Dermatolog Treat. 2008;19:327-332.

.12 Boguniewicz M, Zeichner JA, Eichenfield LF, Hebert AA, Jarratt M, Lucky AW, et al. MAS063DP is effective monotherapy for mild to moderate atopic dermatitis in infants and children: a multicenter, randomized, vehicle-controlled study. $J$ Pediatr. 2008;152:854-859.

.13 Patrizi A, Capitanio B, Neri I, Giacomini F, Sinagra JL, Raone B, et al. A double-blind, randomized, vehicle-controlled clinical study to evaluate the efficacy and safety of MAS063DP (ATOPICLAIR) in the management of atopic dermatitis in paediatric patients. Pediatr Allergy Immunol. 2008;19:619-625.

.14 Eberlein B, Eicke C, Reinhardt HW, Ring J. Adjuvant treatment of atopic eczema: assessment of an emollient containing N-palmitoylethanolamine (ATOPA study). $J$ Eur Acad Dermatol Venereol. 2008;22:73-82. 
.15 Emer JJ, Frankel A, Sohn A, Lebwohl M. A bilateral comparison study of pimecrolimus cream $1 \%$ and a topical medical device cream in the treatment of patients with atopic dermatitis. J Drugs Dermatol. 2011;10:735-743.

.16 Draelos ZD. An evaluation of prescription device moisturizers. J Cosmet Dermatol. 2009;8:40-43.

.17 Miller DW, Koch SB, Yentzer BA, Clark AR, O'Neill JR, Fountain J, et al. An overthe-counter moisturizer is as clinically effective as, and more cost-effective than, prescription barrier creams in the treatment of children with mild-to-moderate atopic dermatitis: a randomized, controlled trial. J Drugs Dermatol. 2011;10:531537.

.18 Ring J, Alomar A, Bieber T, Deleuran M, Fink-Wagner A, Gelmetti C, et al. Guidelines for treatment of atopic eczema (atopic dermatitis) part I. J Eur Acad Dermatol Venereol. 2012;26:1045-1060. 